\title{
Severe intra- and periventricular hemorrhage: role of arteriolosclerosis related to maternal smoke
}

\author{
Luigi Matturri • Donatella Mecchia • Anna M. Lavezzi
}

Received: 10 March 2011 / Accepted: 13 June 2011 /Published online: 9 July 2011

(C) Springer-Verlag 2011

\begin{abstract}
Objective The authors aimed to describe the atherosclerotic lesions of the cerebral arterioles as a substrate of their rupture and bleeding.

Methods The study was performed on the brain of nine Caucasian fetal victims of intra- and periventricular hemorrhage, all grade IV, and nine control cases.

Results In the nine victims of hemorrhage, the arteriolar wall structure was altered, focally transformed into a deposit of amorphous eosinophilic material. Such changes often affected the full thickness of the wall causing rupture and hemorrhage. In eight of these cases and in two victims of the control group, the mothers were heavy cigarette smokers (15-20 cigarettes/day) before and during pregnancy. Conclusion The authors conclude that intra- and periventricular hemorrhage can be ascribed to the toxic effects of prenatal absorption of nicotine.
\end{abstract}

Keywords Intra- and periventricular hemorrhage .

Arteriolosclerosis $\cdot$ Maternal cigarette smoking

\section{Introduction}

Intra- and periventricular hemorrhage (IV-PVH) remains one of the most important problem of preterm neonates, especially during the first 4 days of life, but it can occur at

L. Matturri $(\bowtie) \cdot$ D. Mecchia $\cdot$ A. M. Lavezzi

"Lino Rossi" Research Center for the Study and Prevention of Unexpected Perinatal Death and SIDS-Department of Surgical, Reconstructive and Diagnostic Sciences, University of Milan, Via della Commenda, 19,

20122 Milan, Italy

e-mail: luigi.matturri@unimi.it any time, including prenatally $[1,2]$. The incidence varies from $20 \%$ to $60 \%$ of premature deliveries [3-11] and it has its greatest frequency in the small premature less than 30 weeks of gestation and $1,500 \mathrm{~g}$ birth weight $[12,13]$.

The knowledge on the etiopathogenesis of IV-PVH is still unknown and the anatomopathological investigations in this field are quite neglected. It is also known that the occurrence of atherosclerotic lesions in fetuses of smoking mothers, especially in the arteries of large and medium caliber [14-17]. The aim of this work is to describe the atherosclerotic lesions of the cerebral arterioles, as a substrate of their rupture and bleeding, and to evaluate whether prenatal absorption of nicotine could be related to the pathogenesis of IV-PVH.

\section{Materials and methods}

We studied nine Caucasian fetal victims of IV-PVH, six males and three females, ranging in age from 24 to 39 weeks of gestation, suddenly and unexpectedly deceased. Regarding the fetal weight at death, six fetuses, aged 24-28 gestational weeks $(\mathrm{gw})$, were under $1,000 \mathrm{~g}$; two fetuses, aged 31 and $33 \mathrm{gw}$, respectively, weighed more than 1,000 g and one case (39 gw, at term of gestation) weighed 3,500 g.

According to Papile's classification [5], all the ventricular hemorrhages were grade IV (intraparenchimal and intraventricular hemorrhage). The study included also a control group consisting of nine stillbirths without IV-PVH who are matched for gestational age at death and death weight to each case of IV-PVH group.

Pregnancy had run a normal course in all cases. The mothers of all the fetuses were aged between 25 to 40 years (average age, 32.5 years old). None of the mothers had any significant pathology. While taking the medical history, the 
mothers were asked for information about drugs or alcohol abuse and smoking habit before, during, and after pregnancy. Overall, ten of the 18 mothers were heavy cigarette smokers (15-20 cigarettes/day) before and during pregnancy and eight were nonsmokers (Table 1).

A complete autopsy examination was carried out. The sections of the brain were fixed in $10 \%$ phosphate-buffered formalin, processed and embedded in paraffin. Sections (5 $\mu \mathrm{m})$ were stained with hematoxylin and eosin, Heidenhain's trichrome (Azan), phosphotungstic hematoxylin, and Alcian blue ( $\mathrm{pH}$ 2.5). All cases included in this study were from Lombardy, a highly polluted Italian region, in which the mean $\mathrm{PM}_{2.5}$ and $\mathrm{P}_{10}$ levels are recognized to contribute in a substantial way to perinatal mortality.

\section{Results}

Postmortem examination revealed that in the brain of each case of the study group, a recent hemorrhage, characterized by a large amount of blood, constituted in part by large clots mixed with fluid blood. The affected cerebral hemisphere was larger than the controlateral, so the brain appeared asymmetric. The cerebral circonvolutions were flattened with narrow grooves and the interhemispheric fissure was deflected with the concavity toward the affected side. In each case, the hemorrhage was wide, particularly localized in the basal nuclei, with flooding of the ventricles. The remaining parenchyma was swollen, grayish-white. The other organs and systems were free.

The histological examination revealed extensive hemorrhagic necrosis. The arteriolar wall was often altered. The structure of the wall was focally transformed into a deposit of amorphous eosinophilic material. Such changes sometimes affected the full thickness of the wall causing rupture and hemorrhage. The examination of the cerebral arteries of the Willis polygon, particularly the basilar artery and middle cerebral artery, and of the lenticulo-striate arterioles showed early atherosclerotic lesions (Figs. 1, 2, and 3). These arteries showed a deep structural alteration, often focal, of the tunica media, characterized by a fragmentation of the smooth muscle cells, forming columns arranged perpendicularly to the axis of the tunica media and infiltrating the intima, which was thickened. The increased thickness of the arterial wall also allowed to the reduction in the vessel lumen, which in turn can cause fluctuations in cerebral blood flow, also implicated in the pathogenesis of the hemorrhage. There were also deposits of glycosaminoglycans (Alcian blue positive), while rare monocytes were present. Similar changes were observed particularly in the coronary district, especially in the anterior descending branch of the left coronary artery. In these districts, the alterations were more severe and widespread. In the victims of the control group, all the cerebral vessels showed a normal structure.

A significant relation was evident between maternal smoking and ventricular hemorrhage. In fact, eight of the ten cases with smoking mother were victims of IV-PVH.

Table 1 Case profiles

\begin{tabular}{|c|c|c|c|c|c|c|}
\hline & Sex & Gestational week & Weight (gr) & Mother's age & Smoke & Death diagnosis \\
\hline 1 & M & 24 & 470 & 32 & + & IVH \\
\hline 2 & M & 24 & 485 & 34 & + & IVH \\
\hline 3 & M & 24 & 475 & 33 & - & chorioamnionitis \\
\hline 4 & M & 24 & 480 & 34 & - & chorioamnionitis \\
\hline 5 & $\mathrm{~F}$ & 25 & 515 & 36 & + & $\mathrm{PVH}$ \\
\hline 6 & M & 25 & 540 & 31 & + & IVH \\
\hline 7 & $\mathrm{~F}$ & 25 & 530 & 30 & + & multifocal placental infarcts \\
\hline 8 & M & 25 & 520 & 34 & - & dilated cardiomyopathy \\
\hline 9 & $\mathrm{~F}$ & 26 & 650 & 29 & + & cardiomyopathy \\
\hline 10 & $\mathrm{~F}$ & 27 & 620 & 37 & + & IVH \\
\hline 11 & M & 28 & 705 & 27 & + & $\mathrm{PVH}$ \\
\hline 12 & M & 28 & 700 & 24 & - & severe chorioamnionitis \\
\hline 13 & $\mathrm{~F}$ & 30 & 1000 & 25 & - & congenital heart disease \\
\hline 14 & $\mathrm{~F}$ & 31 & 1010 & 40 & + & IVH \\
\hline 15 & M & 33 & 1250 & 37 & - & cardiomyopathy \\
\hline 16 & M & 33 & 1170 & 25 & - & IVH \\
\hline 17 & M & 39 & 3500 & 36 & + & IVH \\
\hline 18 & M & 40 & 3500 & 34 & - & bronchopneumonia \\
\hline
\end{tabular}




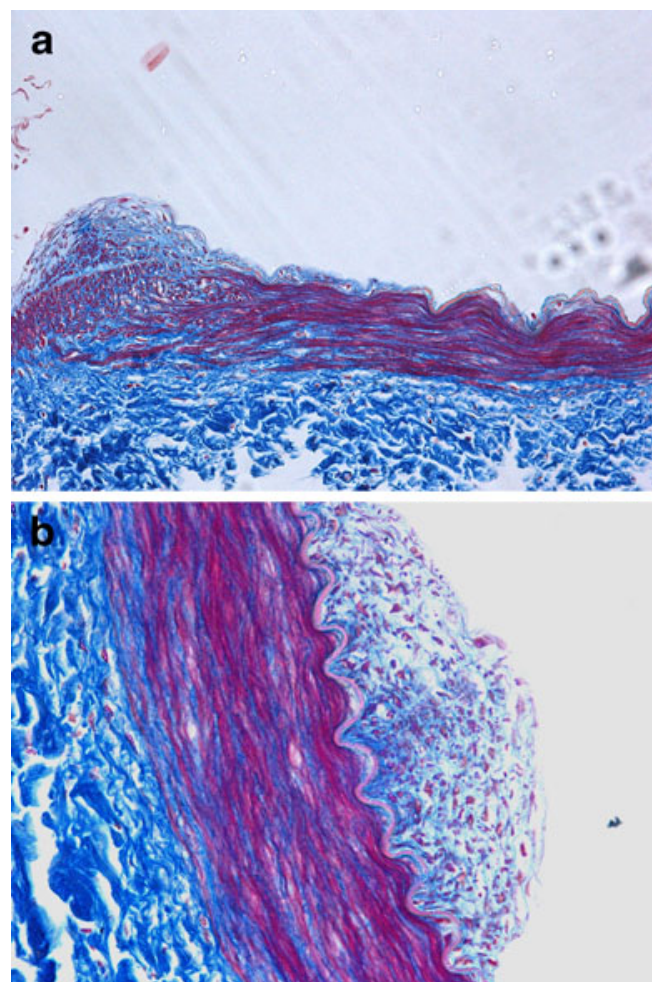

Fig. 1 Basilar artery. Case no. 9, aged 39 gestational weeks. a Focal subversion of the tunica media with fragmentation of the fibers. The myocells form columns, perpendicularly oriented, infiltrating the intima. Azan stain $\times 20$. b The same artery. Thickening of the intima, infiltrated by smooth muscle cells and mast cells. Azan stain $\times 40$

\section{Discussion}

Although the IV-PVH, particularly its association with perinatal factors, has been a subject of several studies [18-23], the cause of this pathology has not yet been explained. An important causal factor is represented by cigarette smoke. Additional predisposing maternal conditions are: bleeding alterations (thrombocytopenia, von Willebrand's disease), drug abuse, seizures, abdominal trauma, and febrile disease [21]. Instead, fetal/neonatal conditions include: immature vessels of the germinal matrix and autoregulation failure of cerebral blood flow in premature, congenital factor $\mathrm{X}$ and factor $\mathrm{V}$ deficiencies, hypoxia-acidosis, twin-twin transfusion, demise of a cotwin, or fetomaternal hemorrhage $[4,21,24,25]$.

The hemorrhage starts in the germinal matrix which is a structure located beneath the ependymal lining, usually between the head of caudate nucleus, adjacent to the foramina of Monroe and the thalamus [18]. This area has a high vascularity and its immature fragile capillary network with poor stromal support, and the sharp "U-turn" the thalamostriate veins, makes the germinal matrix prone to congestion [19]. Arterial supply is provided by the branches of anterior cerebral artery and the venous blood
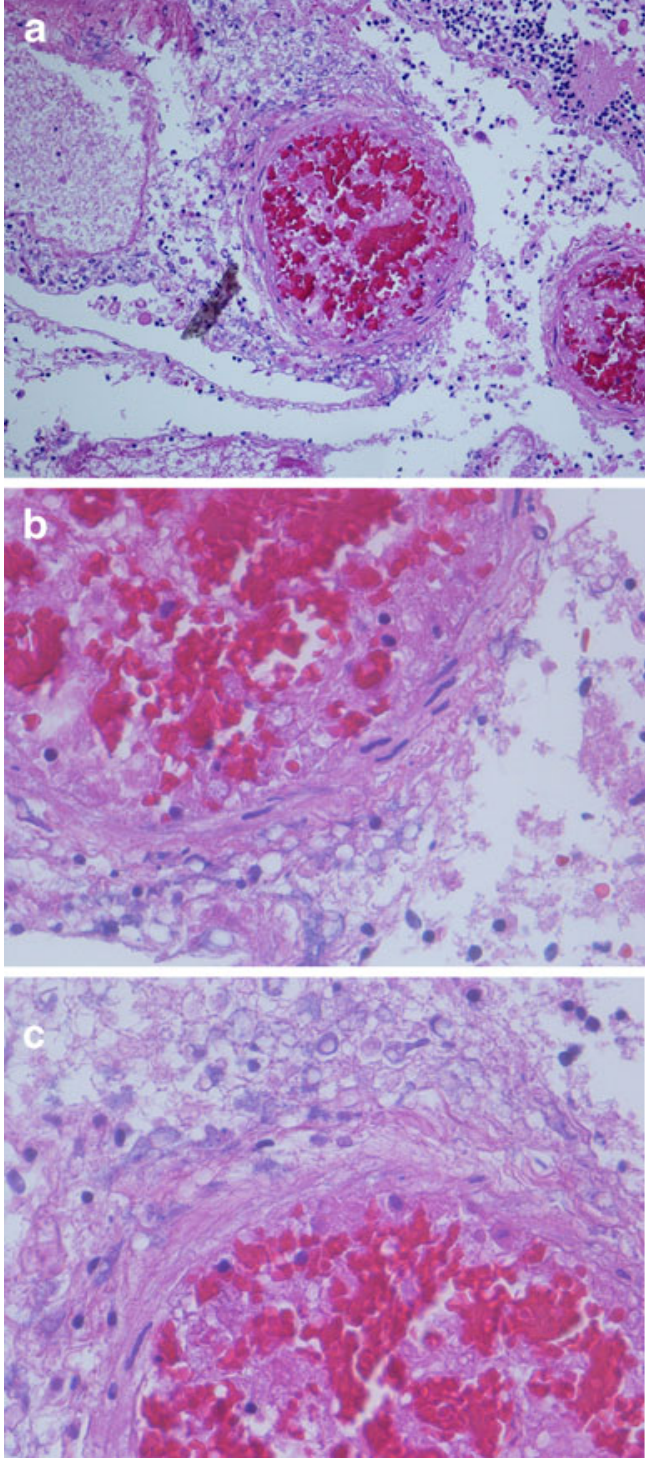

Fig. 2 Lenticulo-striate arteriola. Outbreaks of eosinophilic arteriolonecrosis, next to an area where it is still evident the muscular coat (arrow). a $\times 40$ (zoom). b EE $\times 60$ (particular, normal muscular coat). $\mathbf{c}$ $\mathrm{EE} \times 60$ (particular, eosinophilic arteriolonecrosis)

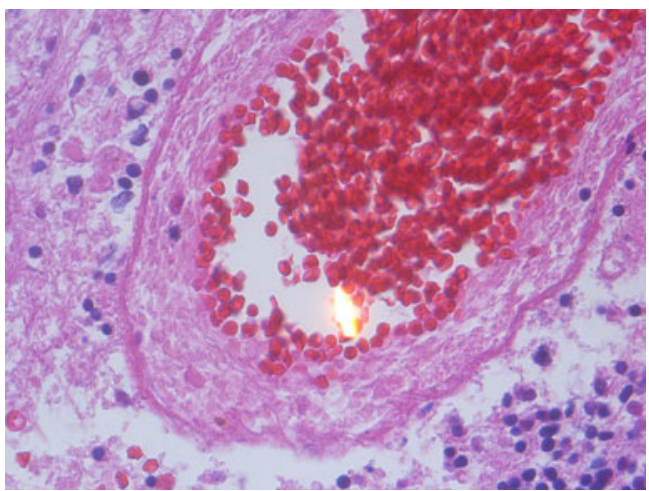

Fig. 3 Lenticulo-striate arteriola. The structure of the arteriolar wall is profoundly altered and transformed into necrotic deposits, homogeneous, and eosinophilic. $\mathrm{EE} \times 60$ 
flow drains through the terminal vein into the vein of Galen [19].

The brain of premature infants has poor ability to autoregulate cerebral blood pressure, so fluctuations in cerebral blood pressure and flow, caused by physical or metabolic insults, can alter the integrity of the thin vessels and lead to bleeding, with or without lateral ventricular involvement, or infarction of the metabolically active germinal matrix [19]. Indeed, this area is a source of both neurons and glial cells during the development of the fetal brain. It is most active between 24 and 32 weeks gestation, then it involutes till 35 weeks and it is much smaller in fullterm infants than prematures [18].

The prognosis and the neurological sequelae are usually related to the severity of the hemorrhage, according to the classification of Papile [18]. Grades 1 and 2 are considered mild and have minimal long-term neurological sequelae; grades 3 and 4 are predictive of severe outcome such as seizures, blindness, mental retardation, cerebral palsy, and death $[20,21]$.

The results of our investigations showed intra- and periventricular hemorrhages, marked by the wide spreading of blood and the frequent involvement of the striated body, characterized by a deep alteration of the wall of the small arteries, lenticulo-optical, and lenticulo-striate. These alterations, which are on the basis of the hemorrhage from rupture, are characterized by a progressive necrosis of the arterial wall. In particular, the tunica media has turned into storage of homogeneous eosinophilic fibrin-like material. These alterations are indeed phenomena of arteriolonecrosis. This tissue, often stuffed with red blood cells, is stained with the methods for fibrin with Azan-Mallory stain (dirty yellow or bright red).

It is important to stress that cerebral arteriolosclerosis is a part of the systemic process of feto-adnexial atherosclerosis $[14,16,17]$. In fact, atherosclerotic lesions in the arteries of various arterial districts are already recognizable in the fetus in the last weeks of gestation, even in the arteries of adnexa. Atherosclerotic lesions are also present in the arteries of medium size, such as those of the conduction system [15].

It is known that pregnant smokers have higher risk for premature delivery and premature rupture of the membranes than nonsmokers and the babies of smoker mothers have greater rates of fetal growth retardation, low birth weight, impaired intellectual development, and neonatal morbidity and mortality [22]. Most of the adverse effects of cigarette smoking are related to chronic hypoxia from decreased uteroplacental perfusion. Besides, nicotine determines significant hemodynamic effects also in the mother by increasing arterial blood pressure and heart rate, which then cause increases of fetal arterial pressure and significant decrease of heart rate and umbilical blood flow [23-26].
These alterations can be traced to the toxic effects of the gaseous components of nicotine, as also demonstrated in large-caliber arteries, arterioles, and in the arteries of adnexa $[18,20,21]$. In addition to the direct hemodynamic effects, toxic effects of the gaseous components released during the combustion of nicotine have been shown to damage the arterial wall $[15,27]$. Therefore, cigarette smoking is an important causal factor of prenatal pathology, also in unexpected and unexplained fetal death [14-17, 2833]. Hence, the strong atherogenic effect of cigarette smoking on human arteries already begins in fetal life, probably in genetically predisposed individuals. This is an important aspect in the prevention of atherosclerosis and its consequences. It is also possible that air pollution, associated with tobacco smoke, may promote and increase its harmful effects.

Acknowledgments The authors thank Dr. Graziella Alfonsi for her precious technical assistance.

Conflict of interest The authors declare no conflict of interest.

\section{References}

1. Tsiantos A, Victorin L, Reiler JP, Dyer N, Sundell H, Brill AB, Stahlman M (1974) Intracranial haemorrhage in the prematurely born infant: timing of clots and evaluation of clinical signs and symptoms. J Pediatr 85:854-859

2. Emerson P, Fujimura M, Howat P, Howes D, Keeling J, Robinson RO, Salisbury D, Tizard JPM (1977) Timing of intraventricular hemorrhage. Arch Dis Child 52:183-187

3. Harke HT, Haeye RI, Storch A et al (1972) Perinatal cerebral hemorrhage. J Pediatr 80:37-42

4. Leech RW, Kohnen P (1974) Subependymal and intraventricular hemorrhages in the newborn. Am J Pathol 77:465-475

5. Papile LA, Burstein J, Burstein R (1978) Incidence and evolution of subependymal and intraventricular hemorrhage: a study of infants with birth weights less than 1,500 gm. J Pediatr 92:529 534

6. Takashima S, Becker LE (1983) Intraventricular and subependymal hemorrhage in infants dying within 10 hours of birth. Brain Dev 5:9-13

7. Philip AG, Allan WC, Tito AM, Wheeler LR (1989) Intraventricular hemorrhage in preterm infants: declining incidence in the 1980's. Pediatrics 84:797-801

8. Jaeger M, Grussner SE, Omwandho CO, Klein K, Tinneberg HR, Klingmuller V (2004) Cranial sonography for newborn screening: a 10-year retrospective study in 11,887 newborns. Rofo 176 :852858

9. Kadri H, Mawla AA, Kazah J (2006) The incidence, timing and predisposing factors of germinal matrix and intraventricular hemorrhage (GMH, IVH) in preterm neonates. Childs Nerv Syst 22:1086-1090

10. Ment LR, Stewart WB, Ardito TA, Madri JA (1995) Germinal matrix microvascular maturation correlates inversely with the risk period for neonatal intraventricular hemorrhage. Development Brain Res 84:142-149

11. Huang YF, Chen WC, Tseng JJ, Ho ESC, Chou MM (2006) Fetal intracranial hemorrhage (fetal stroke): report of four antenatally 
diagnosed cases and review of the literature. Taiwanese J Obstet Gynecol 45:135-141

12. Hack M, Horbar JD, Malloy MH, Tyson JE, Wright E, Wright L (1991) Very low birth weight outcomes of the National Institute of Child Health and Human Development Neonatal Network. Pediatrics 87:587-597

13. Hawgood S, Spong J, Yu VY (1984) Intraventricular hemorrhage. Incidence and outcome in a population of very-low-birth-weight infants. Am J Dis Child 138:136-139

14. Mecchia D, Lavezzi AM, Mauri M, Matturri L (2009) Fetoplacental atherosclerotic lesions in intrauterine fetal demise: role of parental cigarette smoking. Open Cardiovasc Med J 3:51-56

15. Matturri L, Lavezzi AM, Ottaviani G, Rossi L (2005) Early atherosclerotic lesions of the cardiac conduction system arteries in infant. Cardiovasc Pathol 13:276-281

16. Matturri L, Lavezzi AM, Ottaviani G, Rossi L (2003) Intimal preatherosclerotic thickening of the coronary arteries in human fetus of smoker mothers. J Thromb Haemost 1:2234-2238

17. Matturri L, Mecchia D, Lavezzi AM (2009) Birth and progression of systemic atherosclerosis in human fetus and infant of smoker parents. A pathological study. JCCM 4:544-557

18. Burstein J, Papile LA, Burstein R (1979) Intraventricular hemorrhage and hydrocephalus in premature newborns: a prospective study with CT. AJR 132:631-635

19. Donn SM, Bowerman RA (1985) Unilateral germinal matrix hemorrhage in the newborn. J Ultrasound Med 4:251-253

20. Vergani P, Strobelt N, Locatelli A, Paterlini G, Tagliabue P, Parravicini E, Ghidini A (1996) Clinical significance of fetal intracranial hemorrhage. Am J Obstet Gynecol 3:536-543

21. Sherer DM, Anyaegbunam A, Onyeije C (1998) Antepartum fetal intracranial hemorrhage, predisposing factors and prenatal sonography: a review. Am J Perinatol 15:431-441

22. Tejany N, Rebold B, Tuck S, Distroia D, Sutro W, Verma U (1984) Obstetric factors in the causation of early periventricularintraventricular hemorrhage. Obstet Gynecol 64:510-515
23. Thorp J, Jones PG, Clark RH, Knox E, Peabody JL (2001) Perinatal factors associated with severe intracranial hemorrhage. Am J Obstet Gynecol 185:859-862

24. Allan WC, Volpe JJ (1986) Periventricular-intraventricular hemorrhage. Pediatr Clin North Am 36:47-62

25. Lou JC, Lassen NA, Friis-Hansen B (1979) Impaired autoregulation of cerebral blood flow in the distressed newborn infant. $\mathrm{J}$ Pediatr 94:118-121

26. Clark KE, Irion GL (1992) Fetal hemodynamic response to maternal intravenous nicotine administration. Am J Obstet Gynecol 167:1624-1631

27. Quigley ME, Sheehan KL, Wilkes MM, Yen SSC (1979) Effects of maternal smoking on circulating catecholamine level and fetal heart rates. Am J Obstet Gynecol 133:685-690

28. Spinillo A, Ometto A, Bottino R, Piazzi G, Iasci A, Rondini G (1995) Antenatal risk factors for germinal matrix hemorrhage and intraventricular hemorrhage in preterm infants. Europ J Obstet Gynecol 60:13-19

29. Matturri L, Lavezzi AM (2006) Fetal arterial changing in response to maternal cigarette smoking: revisiting the natural history of the earliest stage of atherosclerosis. Curr Cardiol Review 2:255-259

30. Duman N, Oren H, Duman M, Ozkan H (2003) Intracranial hemorrhage and hematuria in a neonate associated with heavy maternal smoking. Turk J Pediatr 45:71-73

31. Lieberman E, Torday J, Barbieri R, Cohen A, Van Vunakis H, Weiss ST (1992) Association of intrauterine cigarette smoke exposure with indices of fetal lung maturation. Obstet Gynecol 79:564-570

32. Strauss A, Kirtz D, Madanlou HD, Freeman RK (1985) Perinatal events and intraventricular/subependymal hemorrhage in the verylow birth weight infant. Am J Obstet Gynecol 151:1022-1027

33. Morales WJ, Koerten J (1986) Obstetric management and intraventricular hemorrhage in very-low-birth-weight infants. Obstet Gynecol 68:35-40 